\title{
DIMENSIONAMENTO EVOLUTIVO DE USINAS HIDROELÉTRICAS
}

\author{
Donato da Silva Filho* \\ donato@sel.eesc.sc.usp.br \\ Adriano A. F. M. Carneiro* \\ adriano@sel.eesc.sc.usp.br \\ * Laboratório de Sistemas de Energia Elétrica - LSEE, Depto. de Engenharia Elétrica \\ Escola de Engenharia de São Carlos, Universidade de São Paulo \\ Av. Trabalhador São-Carlense, 400, CEP 13566-590, São Carlos, SP, BRASIL, +55-16-273-9371
}

\begin{abstract}
Sizing a hydropower plant consists in specifying the main features of the plant that will determine its capacity to generate energy. These features are the minimum and the maximum reservoir storage volumes, the installed capacity, and the design and the rated turbine heads. This paper aims to develop an efficient, flexible and automatic approach to optimally determine the hydropower plant features mentioned above. The usual heuristic methods applied to search for the optimal features of a hydropower plant are replaced by an approach that combines Genetic Algorithms and simulation techniques. A new methodology is introduced to evaluate the economic benefits; the simulation model can use different operation policies for the reservoirs and different stream flow time series. Some initial results show the sensitivity of the benefits in relation to changes in the operation policies.
\end{abstract}

KEYWORDS: Hydroelectric systems, hydroelectric power plants, sizing, reservoir, installed capacity, turbines, optimization, simulation, genetic algorithms, cost-benefit analysis.

\section{RESUMO}

O dimensionamento de uma usina hidroelétrica consiste na especificação das principais características físicas da usina

Artigo Submetido em 15/12/02

1a. Revisão em 18/03/04:

Aceito sob recomendação do Editor Associado

Prof. Dr. Glauco Taranto que determinarão sua capacidade de geração de energia. Estas características são os volumes mínimo e máximo do reservatório, a potência instalada e as quedas de projeto e de referência das turbinas. Este trabalho tem por objetivo desenvolver um modelo de otimização eficiente, flexível e automático para determinar as características mencionadas. Os métodos heurísticos usualmente utilizados na busca dos valores ótimos das características são substituídos por uma combinação entre um Algoritmo Genético e um algoritmo de simulação. Introduz-se uma nova metodologia para cálculo dos benefícios econômicos, consideram-se diferentes políticas de operação e também se cria a possibilidade de realização de estudos com diferentes séries de vazões afluentes. Alguns testes iniciais mostram a sensibilidade dos benefícios em relação às políticas de operação.

PALAVRAS-CHAVE: Sistemas hidroelétricos, usinas hidroelétricas, dimensionamento, reservatório, capacidade instalada, turbinas, otimização, simulação, algoritmos genéticos e análise custo/benefício.

\section{INTRODUÇÃO}

A construção de uma usina hidroelétrica traduz-se na realização de um investimento. A partir do capital utilizado para custear a construção da usina, deseja-se que as receitas operacionais, obtidas com a venda da energia gerada ao longo da vida útil do empreendimento, proporcionem lucros.

Dependendo da concepção do aproveitamento hidroelétrico, o montante necessário para sua construção será diferente, 
bem como sua capacidade de produção de energia e os benefícios econômicos obtidos com sua venda.

Embora o processo de dimensionamento seja complexo, o princípio básico que norteia sua execução é bastante simples: os parâmetros de projeto devem ser determinados de forma que a diferença entre os benefícios e os custos seja máxima.

Os custos de um projeto envolvem aquisição de terras, eventuais relocações de cidades ou vilas, construção das estruturas físicas, compra de equipamentos, juros durante a construção, operação e manutenção, medidas mitigadoras de impactos ambientais, etc. (Fortunato, Araripe Neto et al. 1990).

Os benefícios de uma usina hidroelétrica equivalem aos ganhos energéticos que a obra trará ao sistema no qual ela vai se integrar, medidos pela valorização econômica dos acréscimos de Energia Firme, Energia Secundária e Ponta Garantida. Estes ganhos dependem da evolução da demanda de energia, da disponibilidade hidrológica, das regras de operação que determinam o rateio da carga sistêmica entre as diversas usinas, das restrições de intercâmbio, do custo de combustível das termoelétricas, da valorização do suprimento de ponta, etc.

O principal objetivo deste trabalho é desenvolver uma metodologia de dimensionamento de usinas hidroelétricas flexível para que os diversos parâmetros que podem influenciar as dimensões ótimas de uma usina sejam considerados.

Para tanto, utiliza-se uma técnica de otimização resultante da combinação entre Algoritmos Genéticos e um modelo de simulação da operação de sistemas hidroelétricos de geração de energia (Goldberg 1989; Silva Filho \& Carneiro 1998). Além da combinação destas técnicas, a metodologia proposta possui um esquema flexível para valorização econômica da energia gerada, capacidade de simular a operação do sistema hidroelétrico segundo diferentes políticas de operação e a opção de serem utilizadas diferentes séries de vazões afluentes.

\section{PARÂMETROS DE DIMENSIONAMENTO}

Existem cinco parâmetros principais que definem o dimensionamento energético de uma usina hidroelétrica: níveis máximo e mínimo de armazenagem (N.A.Max e N.A.Min, respectivamente), potência instalada e alturas de queda de referência e de projeto das turbinas (CESP 1988).
O N.A.Max corresponde ao nível máximo do espelho d'água do reservatório ${ }^{1}$. A elevação do N.A.Max aumenta a capacidade de armazenagem do reservatório e a queda líquida da usina, proporcionando ganhos de Energia Firme, Energia Secundária e Potência Garantida. Por outro lado, elevam-se os custos de construção da barragem, vertedor e estruturas principais, além de aumentar a área inundada pelo reservatório e, conseqüentemente, os gastos com aquisição de terras.

O nível mínimo de armazenagem, N.A.Min, é o nível mínimo do espelho d'água do reservatório para o qual a usina pode ser operada. Dado um valor de N.A.Max, a redução do N.A.Min proporciona aumento de volume útil, $x_{\text {útil }}=x_{\text {máx }}-x_{\text {min }}, \quad$ com aumento da capacidade de regularização e Energia Firme. Em contrapartida, ao reduzir o N.A.Min, reduz-se a queda líquida média da usina e, conseqüentemente, a Potência Garantida.

A potência instalada é o nível de motorização da usina e, portanto, estabelece o limite superior para a quantidade de energia que pode ser gerada. Aumentos de potência instalada normalmente elevam os benefícios energéticos, porém aumentam os gastos com a casa de força, estrutura de adução, turbinas, geradores, transformadores, etc.

A Queda de Projeto das turbinas é definida como a queda na qual a turbina apresenta eficiência máxima. Para dimensioná-la utilizam-se resultados de estudos de simulação e determina-se a média ponderada da queda líquida pelos valores de geração. Este valor corresponde à altura de queda na qual a usina é mais solicitada e, portanto, deve apresentar maior rendimento.

A Queda de Referência é definida como a mínima queda líquida para a qual a turbina, com o distribuidor totalmente aberto, consegue suprir o gerador com sua potência instalada. A vazão que passa pela turbina quando ela está submetida à Queda de Referência e o distribuidor está totalmente aberto é chamada de Vazão de Referência; tratase da máxima vazão que pode passar pela turbina. Os valores da queda e da vazão de referência determinam o acoplamento entre as características da turbina e do gerador (Army 1985).

\section{MODELOS DE DIMENSIONAMENTO}

O princípio básico que norteia o dimensionamento de uma usina hidroelétrica é a busca por parâmetros de projeto que maximizem a diferença entre os benefícios e os custos do empreendimento.

\footnotetext{
${ }^{1}$ Determinar N.A.Max traduz-se em definir o volume máximo da usina, $x_{\text {maxx }}$. Da mesma forma, determinar N.A.Min traduz-se em definir o volume mínimo da usina, $x_{\min }$.
} 
O cálculo dos custos pode ser feito através de orçamentos, ainda que aproximados, para cada conjunto de parâmetros de dimensionamento que caracterizem a usina hidroelétrica. Opcionalmente, podem ser utilizadas funções de custo ou tabelas que definam o custo do empreendimento para cada valor de N.A.Max, N.A.Min e potência instalada (Stedinger, Sule et al. 1983). Neste trabalho, assume-se que as quedas das turbinas não influenciam os custos.

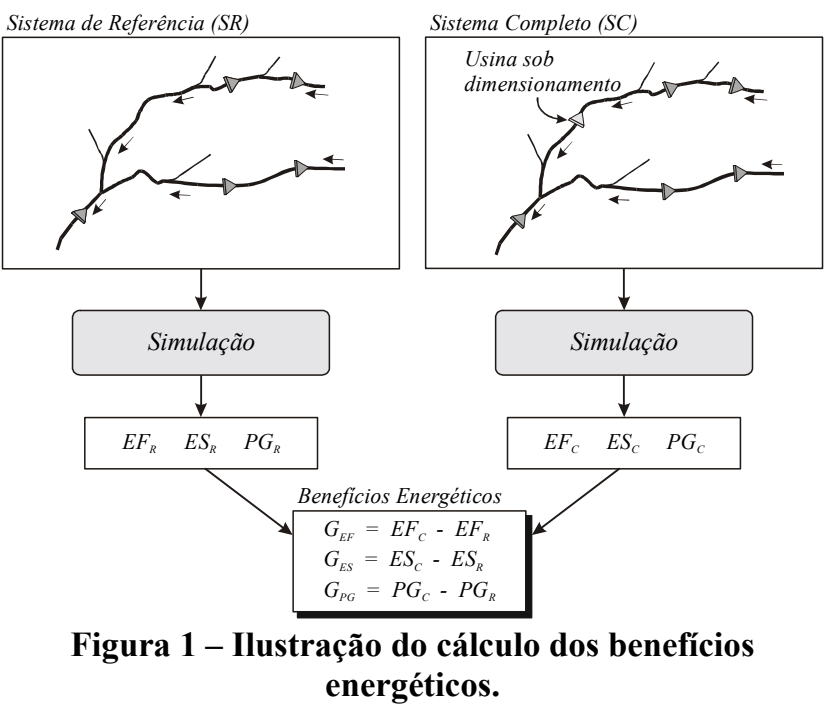

O cálculo dos benefícios é ilustrado na Figura 1. Primeiro, adota-se um Sistema de Referência (SR), definido como o sistema futuro no qual a usina vai inserir-se quando começar a ser operada. Realiza-se a simulação da operação deste sistema e calculam-se seus valores de Energia Firme, Energia Secundária e Ponta Garantida, denotados por $E F_{R}$, $E S_{R}$ e $P G_{R}$, respectivamente. Em seguida, adiciona-se a usina sob dimensionamento ao SR e simula-se a operação do Sistema Completo (SC), determinando os mesmos ganhos energéticos, agora denotados por $E F_{C}, E S_{C}$ e $P G_{C}$. As diferenças entre os valores encontrados caracterizam os ganhos energéticos proporcionados pela usina, denominados $G_{E F}, G_{E S}$ e $G_{P G}$.

Este tipo de abordagem permite que a usina seja valorizada não apenas pela sua geração, mas também pelos ganhos que ela proporciona ao restante do sistema, regularizando melhor as vazões afluentes e permitindo que outras usinas também se beneficiem de sua existência (Silva Filho, Carneiro et al. 2000).

Uma das dificuldades encontradas no processo de dimensionamento é a forma como é realizada a busca por valores ótimos para os parâmetros. Devido à necessidade dos estudos de simulação, adotam-se valores para o conjunto de parâmetros, realiza-se a respectiva simulação e medem-se os benefícios energéticos; com base nos resultados alteram-se os valores dos parâmetros e realiza-se outro estudo de simulação, até que valores "satisfatórios" sejam encontrados. A dificuldade reside em como alterar os parâmetros para que a relação custo/benefício melhore a cada iteração e que o espaço de soluções possíveis seja eficientemente explorado.

O processo de dimensionamento também depende diretamente dos estudos de simulação, pois todos os cálculos dos benefícios energéticos são realizados por simuladores da operação das usinas. Uma das características dos modelos de simulação que pode alterar sensivelmente os estudos de dimensionamento é a política de operação adotada, a qual define a participação de cada usina hidroelétrica no suprimento da carga total do sistema. Dependendo da política de operação, alteram-se os benefícios energéticos das usinas e, conseqüentemente, suas respectivas dimensões ótimas.

Em (Eletrobrás 1997), apresenta-se o Sistema de Inventário Hidroelétrico de Bacias Hidrográficas - SINV. Este sistema tem como objetivo automatizar a execução dos estudos energéticos, econômicos e ambientais, além de permitir a comparação e a seleção de alternativas de divisão de quedas. $\mathrm{O}$ modelo considera como benefício energético apenas a Energia Firme, sem valorizar a Energia Secundária e contabilizando apenas de forma implícita os ganhos de Ponta Garantida. No SINV, o N.A.Max não é submetido a processo de otimização, sendo fixado na definição do aproveitamento. O N.A.Min é definido como o nível mínimo que acrescenta o maior ganho de Energia Firme ao Sistema de Referência. A Queda de Referência é definida como a queda líquida máxima.

Em (Loucks, Stedinger et al. 1981), definem-se modelos para determinação da capacidade de armazenagem de reservatórios. Os modelos referem-se basicamente à minimização do volume útil do reservatório, garantindo-se, com certa confiabilidade preestabelecida, uma vazão regularizada mínima de jusante. Conclui-se que o tamanho final do reservatório depende sensivelmente da política de operação adotada e da seqüência de vazões afluentes utilizada durante o estudo.

Em (Stedinger, Sule et al. 1983), um sistema de suprimento de água composto por três reservatórios é dimensionado com o objetivo de minimizar os custos. Quatro modelos distintos são empregados: (i) VMM - modelo linear baseado em Vazões Médias Mensais, (ii) VPC -modelo linear baseado somente nas Vazões do Período Crítico, (iii) EI -modelo Estocástico Implícito, e (iv) EE - modelo Estocástico Explícito. Os resultados mostram que o VMM, por trabalhar com séries muito uniformes, determina reservatórios muito pequenos. O VPC, por trabalhar com o período mais severo do histórico, superdimensiona os reservatórios. O EI apresenta resultados satisfatórios e o EE mostra-se instável, ora super e ora subdimensionando os reservatórios. 
Em (Sinha, Rao et al. 1999) desenvolve-se um modelo para dimensionamento de reservatórios considerando vários usos da água, incluindo geração de eletricidade. $O$ objetivo também é a minimização dos custos. No trabalho integramse técnicas de otimização e simulação: o modelo de otimização considera apenas as metas anuais de vazão; os valores especificados pelo modelo de otimização são fornecidos ao modelo de simulação, onde avaliam-se as soluções propostas. A integração simulação/otimização produz projetos para o sistema com custos bem inferiores às metodologias tradicionalmente empregadas, onde modelos de simulação são atrelados a buscas heurísticas baseadas na experiência de especialistas. Mostram-se assim os benefícios da criação de métodos que sistematicamente exploram o espaço de soluções.

Em (Bettega \& Ramos 2001) apresenta-se um modelo para análise de viabilidade de usinas hidroelétricas que considera o impacto do mercado spot e Mecanismos de Realocação de Energia (MRE) no cálculo dos benefícios energéticos de um aproveitamento. Os resultados mostram a importância do MRE na viabilidade da usina.

\section{MODELO PROPOSTO}

O modelo proposto combina Algoritmos Genéticos (AGs) e simulação para dimensionar usinas hidroelétricas. $\mathrm{O} A G$ possibilita que o espaço de soluções do problema de dimensionamento seja explorado de forma eficiente. Esta eficiência resume-se a duas características do algoritmo: $(i)$ evitar a exploração exaustiva de cada solução possível, o que seria inviável devido ao tempo necessário para simular cada solução e avaliar os benefícios, (ii) sistematizar a busca de soluções ótimas, evitando a subexploração do espaço de buscas.

O modelo de simulação adotado permite que diferentes políticas de operação sejam avaliadas no processo de dimensionamento. Para o desenvolvimento dos trabalhos iniciais são utilizadas duas políticas de operação: a Regra Paralela (RP) e a Regra baseada em Otimização (RO) (Silva Filho \& Carneiro 1998).

Tanto o algoritmo genético quanto o simulador, implementados para este trabalho, são explicados na seqüência.

\subsection{Algoritmos Genéticos}

Algoritmos Genéticos (AGs) são técnicas de busca baseadas em princípios de evolução e mecanismos de seleção natural e "sobrevivência do mais apto" (Goldberg 1989). Os AGs operam de forma iterativa sobre uma população ou conjunto de soluções do problema a ser otimizado. Estas possíveis soluções representam codificações do problema em uma forma análoga a cromossomos de sistemas biológicos. Há um valor de aptidão associado a cada cromossomo, de forma que quanto melhor a solução que um cromossomo representa, maior a sua aptidão e as suas chances de sobreviver e produzir descendentes.

Geralmente, os AGs possuem dois tipos básicos de operadores: genéticos e evolucionários. Os operadores genéticos, basicamente os operadores de cruzamento e mutação, são responsáveis por determinar como indivíduos vão trocar ou simplesmente alterar suas características genéticas para produzir novos indivíduos. Os operadores evolucionários preocupam-se em determinar quais indivíduos sofrerão cruzamento e mutação (Gen \& Cheng 2000).

A estrutura geral de um AG pode ser descrita como segue, onde $t$ é o índice da geração atual e $P(t)$ é a população de indivíduos da geração $t$.

$-t=0$;

- Inicia a população $P(t)$;

- Avalia a população $P(t)$;

- Enquanto nenhum critério de parada for satisfeito:

- $t=t+1$;

- Aplica-se seleção sobre $P(t-1)$ e gera-se $P(t)$;

- Aplica-se cruzamento sobre $P(t)$;

- Aplica-se mutação sobre $P(t)$;

- Avalia-se $P(t)$;

Como os AGs são técnicas heurísticas relativamente novas, muitos operadores genéticos e evolucionários continuam sendo propostos. Assim, organizar o código computacional dos AGs, fazendo-o altamente estruturado e ao mesmo tempo capaz de incorporar novos operadores, é um grande desafio. Para que o AG deste trabalho apresentasse estas desejáveis características de fácil manutenção e expansão, ele foi desenvolvido em linguagem $\mathrm{C}++$, seguindo $\mathrm{o}$ paradigma de programação orientada por objetos (Stroustrup 2000).

A implementação de AG utilizada, desenvolvida inicialmente para este trabalho, é chamada GOOAL Genetic Object Oriented ALgorithm (Algoritmo Genético Orientado por Objetos). A estrutura geral do GOOAL, representada através de seu diagrama de classes, é mostrada na Figura 2. A classe principal, responsável por fazer todas as outras trabalharem juntas, é a TGOOAL. Esta classe contém objetos das outras classes, tais como TCruzamento, TMutacao, TPopulacao, TEscalonamento, TSelecao, TEstrutura, TParada, TElitismo e TImpressao. 


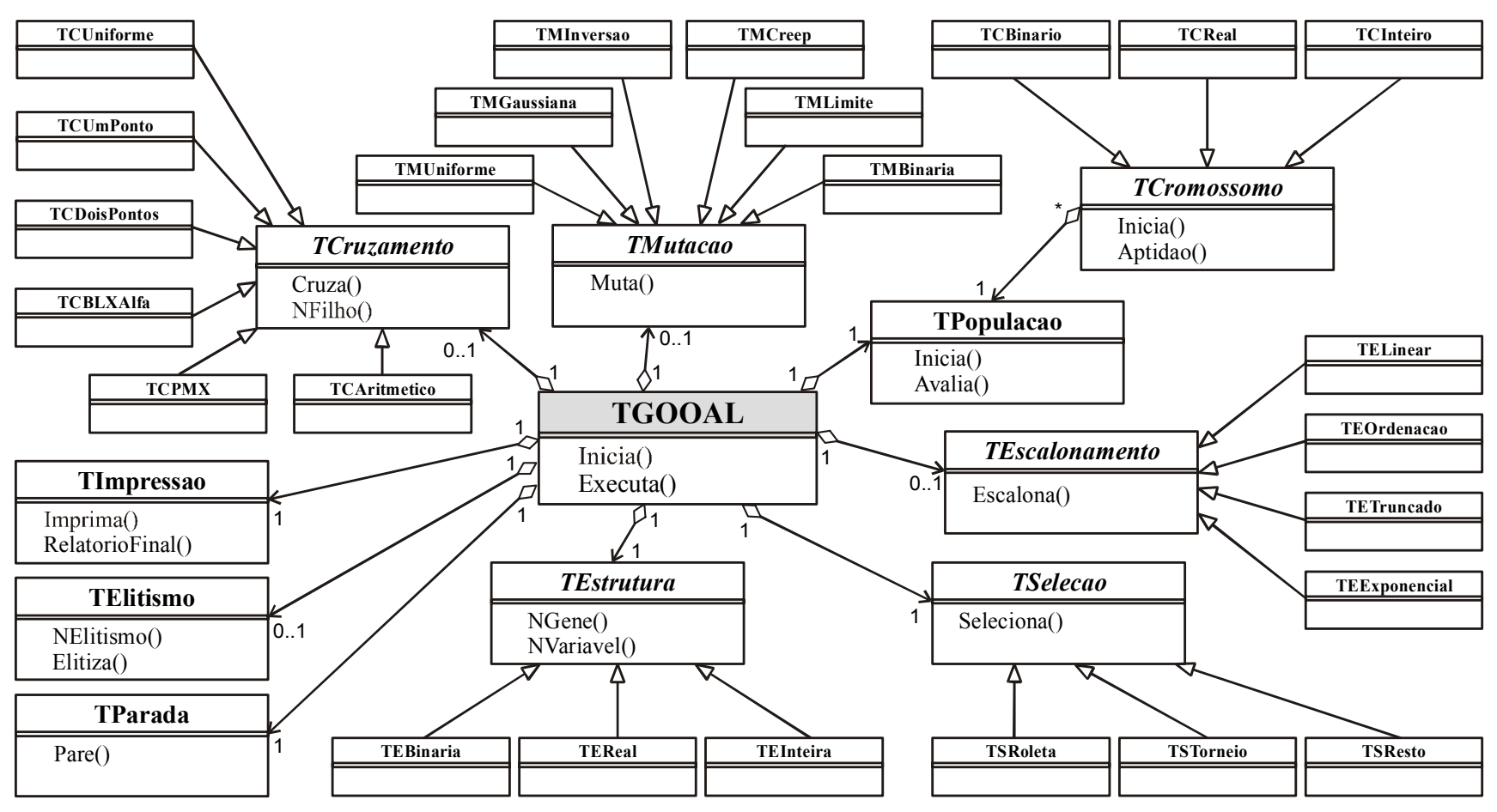

Figura 2 - Diagrama de classes do GOOAL.

Os nomes das classes auto-sugerem seus propósitos. Por exemplo, a classe TCruzamento implementa os operadores de cruzamento. Como há diversos operadores de cruzamento possíveis, a classe TCruzamento é implementada como abstrata, ou seja, ela apenas estabelece quais métodos os operadores de cruzamento devem ter: Cruza() e Nfilho() (Horstmann 1997).

As classes que realmente combinam indivíduos são aquelas implementadas como diferentes tipos de TCruzamento, usando mecanismos de herança. Estas classes são a TCUniforme, TCUmPonto, TCDoisPontos, etc. A classe TCUniforme realiza o cruzamento uniforme entre dois indivíduos através de seu método Cruza(), a classe TCUmPonto realiza o cruzamento de um ponto, e assim por diante.

As classes TCromossomo e TEstrutura também estabelecem padrões, mas nesse caso elas estão focadas na estruturação do algoritmo. A classe TEstrutura define como os dados são alocados na memória. Por exemplo, o número de genes de uma representação binária que codifica três variáveis depende da precisão adotada, enquanto que para a representação real, o número de genes é igual ao número de variáveis. Desta forma, diferentes métodos NGene() são implementados para as classes TEBinario, TEReal e TEInteiro. Similarmente, a classe TCromossomo apresenta comportamentos diferenciados dependendo do tipo de representação: binária, real ou inteira.
Outras classes desenvolvem seus papéis sem a necessidade de mecanismos de especialização. Estas classes são a TImpressao, que imprime relatórios sobre o processo de busca, TElitismo, que copia os melhores cromossomos de uma geração a outra, e a TParada, que decide quando o processo de busca deve parar.

Todas as classes brevemente descritas são as mesmas, independentemente do problema a ser resolvido. O que faz uma aplicação do GOOAL ser diferente de outra é a função que determina a aptidão de cada cromossomo. A função de avaliação para o dimensionamento de uma usina hidroelétrica é mostrada na seção 4.4. Antes são mostrados o modelo de simulação e a codificação adotada.

\subsection{Algoritmo de Simulação}

$\mathrm{O}$ algoritmo de simulação utilizado baseia-se em regras de enchimento/esvaziamento dos reservatórios, ou simplesmente regras de operação. Neste estudo, por operação do sistema entende-se a decisão de geração e conseqüente operação dos reservatórios num horizonte de longo prazo, com intervalos de tempo mensais (Carneiro \& Soares 1993).

Sob o ponto de vista da operação, somente as usinas com reservatório de acumulação podem variar a quantidade de água armazenada em seus reservatórios. As usinas a fio 
d'água mantêm armazenagem constante, com vazão defluente igual à afluente.

A regra de operação classicamente adotada pelo setor elétrico brasileiro é a Regra Paralela (RP), onde as usinas definem suas vazões turbinadas de forma a manterem iguais percentuais de volume nos reservatórios de acumulação. Definindo $\lambda$ como sendo o fator de acoplamento entre as usinas, com $0 \leq \lambda \leq 1$, a regra paralela pode ser escrita conforme a Equação 1.

$$
x(\lambda)=x_{\min }+\lambda \cdot\left(x_{\max }-x_{\min }\right)
$$

sendo:

- $\quad x(\lambda)$ : volume determinado em função do fator de acoplamento, em $\mathrm{hm}^{3}$;

- $x_{\min }$ : volume mínimo operativo da usina, em $\mathrm{hm}^{3}$;

- $x_{\max }$ : volume máximo operativo da usina, $\mathrm{em} \mathrm{hm}^{3}$.

Para que as regras de operação incorporem critérios de otimização, procura-se fazer com que expressem, de alguma forma, o comportamento operativo ótimo dos sistemas. Para tanto, escolheu-se um fator de acoplamento relacionando o estado de cada reservatório com a energia armazenada no sistema total. Estabelece-se assim uma relação física entre as variáveis a serem utilizadas na regra: o dado de entrada é a energia armazenada no sistema e a resposta, determinada pela regra, é o volume de cada reservatório. Esta nova regra, mostrada em (2), pode ser entendida como uma generalização de (1).

$$
x(\lambda)=x_{\min }+f_{i}(\lambda) \cdot\left(x_{\max }-x_{\min }\right)
$$

As funções $f_{i}(\lambda)$ expressam as regras de operação obtidas com base nos resultados da otimização. Inicialmente, $\mathrm{o}$ sistema completo é otimizado sobre as mais diversas condições hidrológicas possíveis. Em seguida, ajustam-se curvas sobre as nuvens de pontos criadas, plotando-se o volume armazenado de cada usina em função da energia armazenada no sistema. As curvas ajustadas correspondem às funções $f_{i}(\lambda)$.

Na Figura 3 apresenta-se um exemplo com algumas regras de operação para quatro usinas em cascata: Emborcação, Itumbiara, São Simão e Ilha Solteira (vide Figura 8). Para obter as nuvens de pontos, foram realizados estudos de otimização da operação destas usinas entre os anos de 1931 e 2000 (Carvalho \& Soares 1987). As regras foram ajustadas com base em conceitos de Lógoca Nebulosa (Silva Filho \& Carneiro 1998).

Pelo formato das curvas, ao reduzir-se o fator de acoplamento do valor máximo (1) até zero, nota-se que as

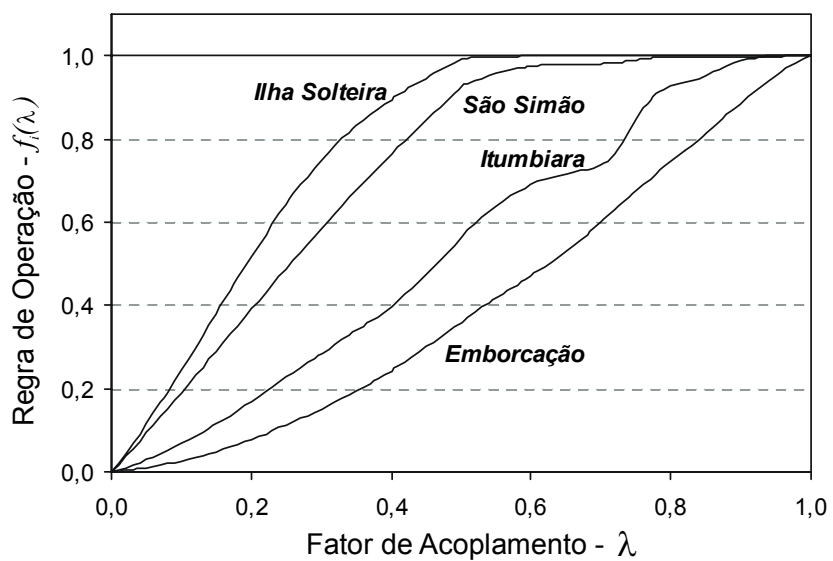

Figura 3 - Regras de operação para algumas usinas em cascata.

regras baseadas em otimização (ROs) determinam que Ilha Solteira e São Simão permaneçam cheias a maior parte do tempo; que Emborcação esvazie-se tão $\operatorname{logo} \lambda$ seja reduzido, e que Itumbiara apresente um comportamento intermediário. Nota-se, portanto, que o uso a solução ótima na obtenção de regras de operação de reservatórios apresenta resultados bem diferentes daqueles encontrados com a regra paralela, onde todos os reservatórios enchem-se e esvaziam-se ao mesmo tempo. De acordo com a RO, as usinas de jusante devem tentar permanecer cheias, mantendo alta produtividade, valorizando assim os grandes volumes de água que fluem por elas. Já as usinas de montante devem ser responsáveis pela regularização das vazões afluentes, amortecendo os picos de vazão e evitando vertimentos nas usinas que estão abaixo. O modelo de simulação, ilustrado na Figura 4, pode ser explicado de forma simplificada em nove passos: (1) fornecimento de dados de vazões afluentes, volumes dos reservatórios no mês anterior e meta de geração para o estágio atual; (2) atribuição de um valor inicial a $\lambda$, dividindo a energia armazenada ao final do intervalo $t-1$ pela energia armazenada máxima no sistema; (3) cálculo dos volumes dos reservatórios utilizando-se as regras de operação e o valor de $\lambda$; (4) determinação da vazão defluente de cada usina realizando-se o balanço d'água; (5) cálculo dos limites inferior e superior de vazão turbinada, volume armazenado e geração de cada usina; (6) resolução de conflitos, compatibilizando as decisões da política de operação com as restrições operativas; (7) cálculo da geração de cada usina e do sistema; (8) comparação da geração calculada com a meta desejada; (9) verificação de convergência, podendo-se alterar o valor de $\lambda$ caso a meta ainda não tenha sido atendida. 


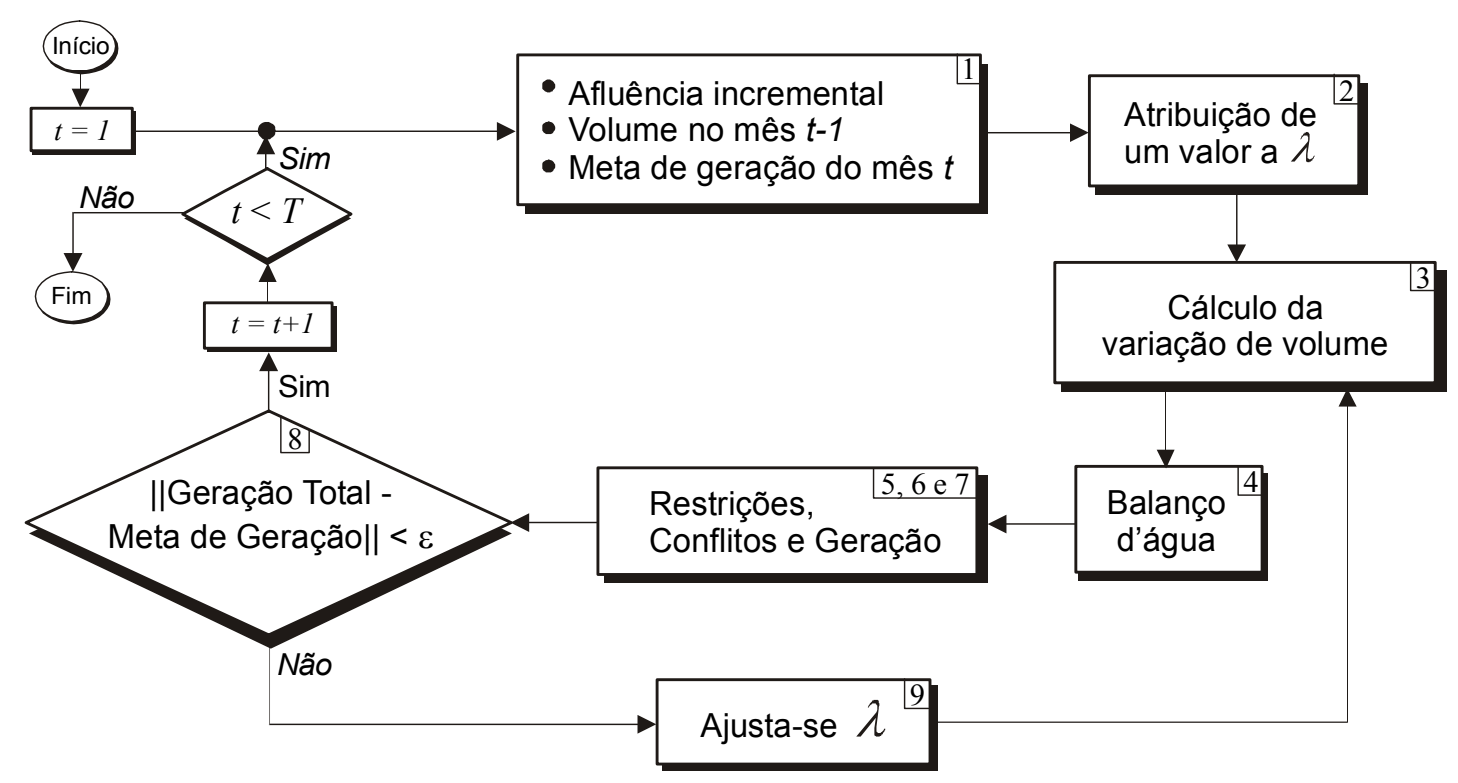

Figura 4 - Fluxograma do algoritmo de simulação.

A variação de $\lambda$ dá-se da seguinte forma:

- Excesso de geração: se a geração hidroelétrica total é maior que o mercado $\mathrm{D}$, eleva-se $\lambda$ de forma a armazenar o excesso de água e reduzir a vazão turbinada, com conseqüente redução de geração. Se $\lambda$ igualar-se a um, indicando que todos os reservatórios estão cheios, e ainda houver excesso de água, este excesso é vertido.

- Déficit de geração: se a geração hidroelétrica total é menor que o mercado, reduz-se $\lambda$ de forma a utilizar a água dos reservatórios e elevar a vazão turbinada, com conseqüente elevação da geração. Se $\lambda$ igualar-se a zero, indicando que todos os reservatórios estão vazios, e a geração total do sistema ainda for menor que o mercado, caracteriza-se um déficit de suprimento de energia.

\subsection{Codificação Utilizada}

Para a aplicação do AG no dimensionamento de usinas hidroelétricas, o problema foi codificado de forma que os cromossomos representem as variáveis de decisão, tal como ilustrado na Figura 5.

\begin{tabular}{|c|c|c|c|}
\hline$x_{l}$ & $x_{2}$ & $x_{3}$ & $x_{4}$ \\
\hline 010 & $\cdots 000$ & 011. & 100 \\
\hline & & $\begin{array}{c}q_{\text {nom }} \\
\text { Vazão dereferencia } \\
\text { nominal }\end{array}$ & $\begin{array}{c}\text { Queda de referêen } \\
\text { nominal }\end{array}$ \\
\hline
\end{tabular}

Figura 5 - Esquema de um cromossomo.
Para codificar os volumes mínimo e máximo do reservatório, são fornecidos dois limites, $x f_{\min }$ e $x f_{\max x}$, que delimitam a faixa de valores possíveis do volume máximo. Utiliza-se a seguinte codificação:

- $x_{1}$ : primeira variável do cromossomo, representa o volume mínimo do reservatório, pertencente ao intervalo $\left[x f_{\min }\right.$, $\left.x f_{\text {máx }}\right]$;

- $x_{2}$ : segunda variável do cromossomo, representa o volume máximo do reservatório, pertencente ao intervalo $\left[x_{1}, x f_{\text {máx }}\right]$;

A terceira variável do cromossomo, $x_{3}$, é a vazão de referência das turbinas, $q_{\text {nom }}, \mathrm{em} \mathrm{m}^{3} / \mathrm{s}$. Seus limites mínimo e máximo, $q_{\text {nom,min }}$ e $q_{\text {nom,max }}$, são determinados a partir da análise da distribuição de vazões do conjunto de vazões fornecido.

Finalmente, a quarta variável do cromossomo, $x_{4}$, é a queda de referência nominal das turbinas, $h_{\text {nom }}$, em $m$. Seus limites mínimo e máximo, $h_{\min }$ e $h_{\text {máx }}$, são determinados com base nos volumes mínimo e máximo, da seguinte forma:

$$
\left\{\begin{array}{l}
h_{\text {min }}=h_{\text {mon }}\left(x_{1}\right)-c f_{\text {med }}-p c_{\text {méd }} \\
h_{\text {max }}=h_{\text {mon }}\left(x_{2}\right)-c f_{\text {med }}-p c_{\text {méd }}
\end{array}\right.
$$

na qual:

- $h_{m o n}(x)$ : polinômio que especifica o nível de montante, em $m$, em função do volume $x$, em $\mathrm{hm}^{3}$;

- $c f_{\text {méd }}$ nível médio do canal de fuga, em $m$;

- $p c_{\text {méd }}$ : perda de carga média, em $m$. 
Com base nos valores de $x_{3}$ e $x_{4}$, determina-se a potência nominal dos geradores, em MW:

$$
p_{\text {nom }}=9,81 \cdot 10^{-3} \cdot \eta_{\text {med }} \cdot x_{4} \cdot x_{3}
$$

onde $\eta_{\text {méd }}$ é o rendimento médio do conjunto turbinagerador.

A queda de projeto das turbinas é definida após a convergência do algoritmo genético, utilizando os resultados da simulação da usina com dimensões ótimas.

\subsection{Função de Avaliação}

Cada cromossomo da população representa características da usina hidroelétrica sob dimensionamento. Para calcular o valor da aptidão de um cromossomo, seguem-se os passos descritos na Figura 6.

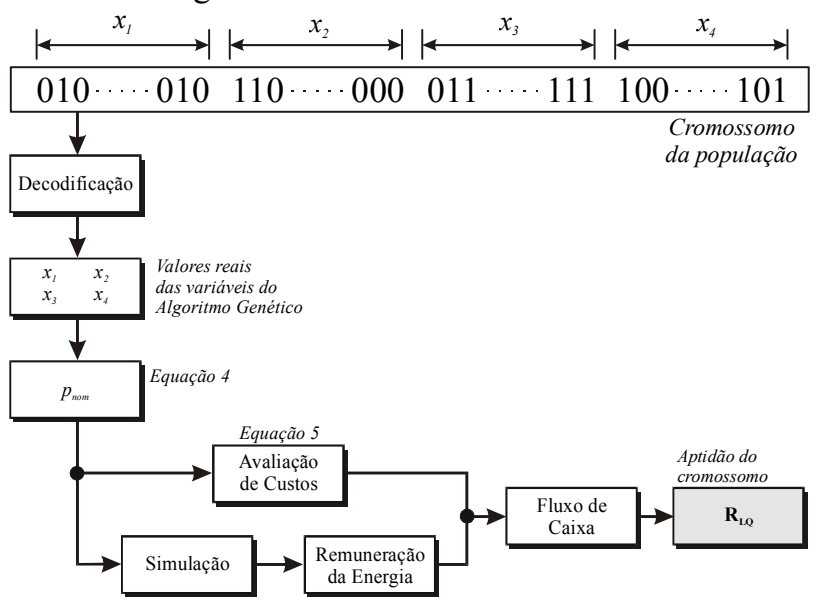

Figura 6 - Cálculo da aptidão de um cromossomo.

Primeiro, quando o cromossomo é decodificado, obtém-se os valores das variáveis $x_{1}, x_{2}, x_{3}$ e $x_{4}$. Utilizando (4), determina-se a potência nominal, $p_{\text {nom }}$.

Com todos estes valores disponíveis, seguem-se dois caminhos paralelos: no primeiro, calculam-se os custos; no segundo, simula-se a operação do Sistema Completo e avaliam-se os benefícios econômicos.

Os dois caminhos paralelos encontram-se no cálculo de um fluxo de caixa, ilustrado na Figura 7. Os parâmetros da figura são os seguintes:

- Tempo de construção da obra, $T_{C}$, em anos;

- Vida útil da usina, $T_{U}$, em anos;

- Cronograma de desembolso, sendo $D_{j}$ a parcela paga no ano $j, 0 \leq j \leq T_{C}$;

- Taxa de juros durante a construção, $t_{j d c}$.

- Taxa de desconto, $t_{d}$.
As funções de custo determinam o investimento total, $I_{T}$, necessário para construir a usina:

$$
I_{T}=c_{c r}\left(x_{\text {max }}\right)+a e\left(h_{\text {mon }}\left(x_{\text {max }}\right)\right) \cdot c_{a t}\left(x_{x \max }\right)+c_{g t}\left(p_{\text {nom }}\right)
$$

onde:

- $c_{c r}\left(x_{2}\right)$ : custo de construção do reservatório como função do volume máximo, em $\mathrm{hm}^{3}$;

- $a e\left(h_{m o n}\left(x_{2}\right)\right)$ : área do espelho d'água, em $\mathrm{km}^{2}$, em função do nível de montante para o reservatório cheio, em $m$;

- $c_{a t}\left(x_{\text {máx }}\right)$ : custo de aquisição de um $\mathrm{km}^{2}$ de terra como função do volume máximo, em $\mathrm{hm}^{3}$;

- $c_{g t}\left(p_{\text {nom }}\right)$ : custo dos geradores e das turbinas como função da potência instalada, em MW.

O valor do investimento total é transformado em parcelas de acordo com o esquema de desembolso. Deve-se garantir a seguinte relação:

$$
I_{T}=\sum_{j=0}^{T_{C}} D_{j}
$$

Para calcular a atratividade econômica do empreendimento, calcula-se o valor presente da receita líquida, $R_{L Q}$, referido ao início do ano de entrada em operação da usina, dado por (7).

$$
R_{L Q}=R_{V P}-D_{V P}-C_{C O M, V P}
$$

onde $R_{V P}$ o valor presente das receitas, $D_{V P}$ o valor presente dos desembolsos e $C_{C O M, V P}$ o valor presente dos custos de operação e manutenção.

A receita líquida do empreendimento é utilizada como aptidão do cromossomo. Eventualmente, se $R_{L Q}<0$, faz-se $R_{L Q}=0$.

\subsection{Resultados do Modelo}

O resultado principal do modelo é o conjunto de dimensões ótimas da usina sob dimensionamento. Assim, o melhor indivíduo encontrado pelo Algoritmo Genético é

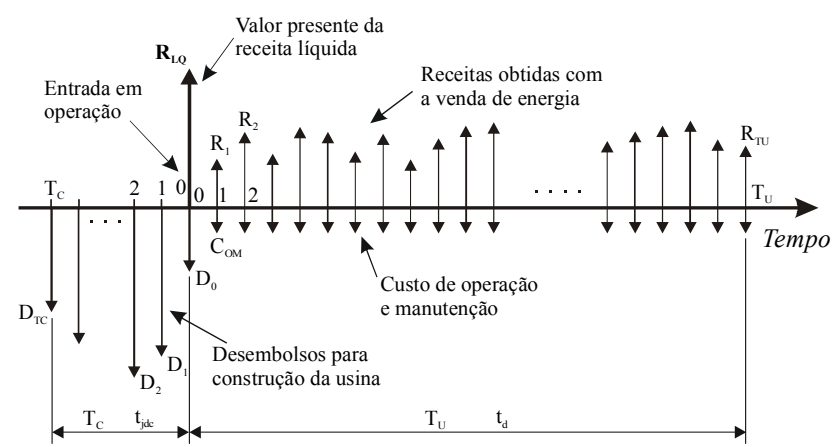

Figura 7 - Fluxo de caixa do investimento. 
decodificado e obtêm-se os valores dos parâmetros de dimensionamento.

Adicionalmente, o modelo também fornece as mudanças que a construção da usina proporciona nas outras usinas do sistema, mostrando os benefícios energéticos de cada usina, antes e depois da construção do novo empreendimento. Eventualmente, algumas usinas beneficiam-se e outras acabam sendo prejudicadas.

\section{TESTES INICIAIS}

Os primeiros testes realizados com o modelo visam determinar como as políticas de operação podem influenciar os benefícios energéticos do aproveitamento sob dimensionamento.

Adota-se como Sistema Teste as principais usinas do Sistema Sudeste, mostradas na Figura 8, e supõe-se que algumas usinas já existentes estejam sendo dimensionadas.

Analisam-se os seguintes parâmetros de dimensionamento: volume máximo, potência instalada e quedas das turbinas. As variações de volume máximo são analisadas em Emborcação e Itumbiara; as variações de potência instalada são avaliadas em Itumbiara e São Simão; e os parâmetros das turbinas são estudados somente em Itumbiara.

As variações dos parâmetros foram sempre avaliadas através de três valores (vide tabelas da próxima página), onde o valor intermediário corresponde à dimensão real da usina. Os outros dois valores foram calculados de forma a permitir que os benefícios energéticos sentissem suas variações e, ao mesmo, a factibilidade dos modelos matemáticos das usinas fosse mantida (limites dos polinômios cota $x$ vazão e cota $x$ volume).

Para realização dos estudos com uma determinada usina segue-se a seguinte seqüência:

1. Define-se como SR, o Sistema Teste citado, sem a usina sob dimensionamento.

2. Simula-se a operação do SR com a RP e avaliam-se os benefícios energéticos.

3. Insere-se a usina que está sob dimensionamento ao Sistema de Referência e realizam-se então simulações do SC, também com a RP, uma para cada valor do parâmetro de dimensionamento.

4. Comparando os resultados dos passos 2 e 3, obtém-se como os valores do parâmetro de dimensionamento alteram os benefícios energéticos quando a regra de operação é a RP.

5. Repetem-se os passos 2, 3 e 4 para a RO.

6. Comparando os resultados das duas regras têm-se como a política de operação altera o benefício gerado pela modificação estrutural da usina.

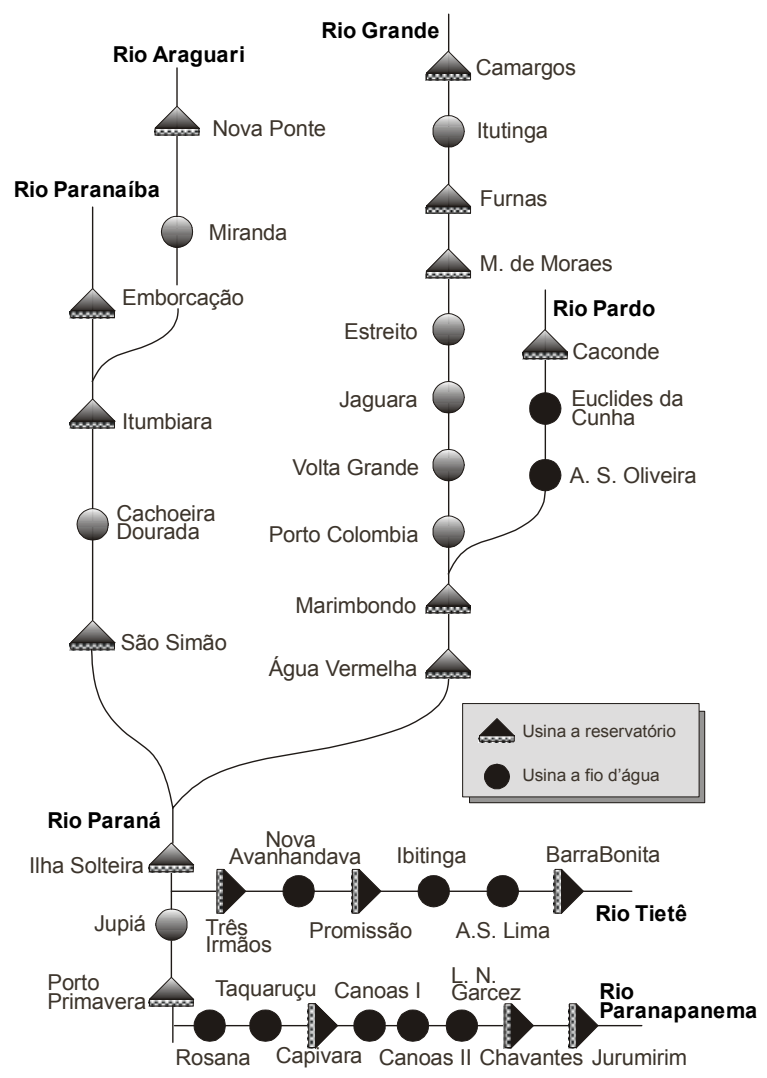

Figura 8 - Sistema teste.

\subsection{Variações de Volume Máximo}

Na Tabela 1 são apresentados os valores de Energia Firme do estudo envolvendo a usina de Emborcação com a RP e três valores distintos de volume máximo.

Na coluna Sistema apresentam-se os valores de Energia Firme do sistema em cada situação. A coluna Dif. Ref. mostra a diferença entre o benefício energético do $\mathrm{SC}$ e do $\mathrm{SR}$, e a coluna Dif. Inc. apresenta os benefícios incrementais proporcionados pela alteração do volume máximo.

Através dos valores apresentados, verifica-se que a Energia Firme do Sistema de Referência com a RP é 10 552,7 $M W$.mês. A inserção de Emborcação com seus diferentes volumes máximos proporciona ganhos diferenciados. À medida que o reservatório aumenta, os ganhos também aumentam, pois além de haver mais água disponível para geração durante o Período Crítico, o nível de montante da usina também eleva-se.

Na Tabela 2 são apresentados os benefícios energéticos para as mesmas situações da Tabela 1, porém utiliza-se a RO. Comparando as duas tabelas nota-se que para cada valor de volume máximo, os valores de Energia Firme do Sistema são maiores na Tabela 2, ou seja, com as mesmas 
usinas e com as mesmas vazões, a RO consegue gerar mais energia.

Para o caso em que o reservatório de Emborcação aumenta de $13820 \mathrm{hm}^{3}$ para $17190 \mathrm{hm}^{3}$, conclui-se que o benefício energético incremental deste aumento de volume é de 93,5 $M W$.mês com a RP. Já para a $\mathrm{RO}$, o benefício incremental é de 105,8 MW.mês.

Tabela 1 - Energia Firme do sistema em função do volume máximo de Emborcação - RP.

\begin{tabular}{|l|c|c|c|}
\cline { 2 - 4 } \multicolumn{1}{c|}{} & \multicolumn{3}{c|}{ Energia Firme-MW.mês } \\
\multicolumn{1}{c|}{ Sistema } & Dif. Ref. & \multicolumn{1}{c|}{ Dif. Incr. } \\
\hline Sist. Referência & 10552,7 & - & - \\
\hline$x_{\text {máx }}=13820 \mathrm{hm}^{3}$ & 11204,6 & 651,9 & - \\
\hline$x_{\text {máx }}=17190 \mathrm{hm}^{3}$ & 11298,1 & 745,4 & 93,5 \\
\hline$x_{\text {máx }}=20440 \mathrm{hm}^{3}$ & 11384,1 & 813,4 & 68,0 \\
\hline
\end{tabular}

Tabela 2 - Energia Firme do sistema em função do volume máximo de Emborcação - RO.

\begin{tabular}{|l|c|c|c|}
\cline { 2 - 4 } \multicolumn{1}{c|}{} & \multicolumn{3}{c|}{ Energia Firme - MW.mês } \\
\multicolumn{1}{c|}{ Sistema } & \multicolumn{1}{c|}{ Dif. Ref. } & \multicolumn{1}{c|}{ Dif. Incr. } \\
\hline Sist. Referência & 10766,9 & - & - \\
\hline$x_{\text {máx }}=13820 \mathrm{hm}^{3}$ & 11441,0 & 674,1 & - \\
\hline$x_{\text {máx }}=17190 \mathrm{hm}^{3}$ & 11546,8 & 779,9 & 105,8 \\
\hline$x_{\text {máx }}=20440 \mathrm{hm}^{3}$ & 11679,6 & 912,7 & 132,8 \\
\hline
\end{tabular}

Tabela 3 - Energia Firme do sistema em função do volume máximo de Itumbiara - RP.

\begin{tabular}{|l|c|c|c|}
\cline { 2 - 4 } \multicolumn{1}{c|}{} & \multicolumn{3}{c|}{ Energia Firme - MW.mês } \\
\cline { 2 - 4 } \multicolumn{1}{c|}{ Sistema } & \multicolumn{1}{c|}{ Dif. Ref. } & \multicolumn{1}{c|}{ Dif. Incr. } \\
\hline$x_{\text {máx }}=12700 \mathrm{hm}^{3}$ & 11179,2 & 973,3 & - \\
\hline$x_{\text {máx }}=17027 \mathrm{hm}^{3}$ & 11298,0 & 1092,1 & 118,8 \\
\hline$x_{\text {máx }}=21350 \mathrm{hm}^{3}$ & 11395,7 & 1189,8 & 97,7 \\
\hline
\end{tabular}

Tabela 4 - Energia Firme do sistema em função do volume máximo de Itumbiara - RO.

\begin{tabular}{|l|c|c|c|}
\cline { 2 - 4 } \multicolumn{1}{c|}{} & \multicolumn{3}{c|}{ Energia Firme - MW.mês } \\
\multicolumn{1}{c|}{ Sistema } & Dif. Ref. & Dif. Incr. \\
\hline Sist. Referência & 10395,4 & - & - \\
\hline$x_{\text {máx }}=12700 \mathrm{hm}^{3}$ & 11415,2 & 1019,8 & - \\
\hline$x_{\text {máx }}=17027 \mathrm{hm}^{3}$ & 11546,8 & 1151,4 & 131,6 \\
\hline$x_{\text {máx }}=21350 \mathrm{hm}^{3}$ & 11685,9 & 1290,5 & 139,1 \\
\hline
\end{tabular}

Quando o volume de Emborcação é alterado de $17190 \mathrm{hm}^{3}$ para $20440 \mathrm{hm}^{3}$, pela RP o benefício energético incremental é 68,0 MW.mês, enquanto que para RO ele é 132,8 MW.mês. Há uma diferença de 95\% entre estes dois valores.

Estas alterações nas variações incrementais dos benefícios energéticos implicam em variações de receita líquida, que por suas vezes podem alterar as características ótimas do aproveitamento. Estes testes iniciais tentam justamente mostrar estes efeitos das políticas de operação sobre o cálculo da aptidão mostrado na Figura 6.

Tabela 5 - Energia Firme do sistema em função da potência instalada de Itumbiara - RP.

\begin{tabular}{|l|c|c|c|}
\cline { 2 - 4 } \multicolumn{1}{c|}{} & \multicolumn{3}{c|}{ Energia Firme - MW.mês } \\
\multicolumn{1}{c|}{ Sistema } & Dif. Ref. & \multicolumn{1}{c|}{ Dif. Incr. } \\
\hline$P_{\text {inst }}=1700 \mathrm{R}$. & 10205,9 & - & - \\
\hline$P_{\text {inst }}=2100 M W$ & 11298,1 & 1092,2 & - \\
\hline$P_{\text {inst }}=2500 M W$ & 11298,1 & 1092,2 & 0,0 \\
\hline
\end{tabular}

Tabela 6 - Energia Firme do sistema em função da potência instalada de Itumbiara - RO.

\begin{tabular}{|l|c|c|c|}
\multicolumn{1}{c|}{} & \multicolumn{3}{c|}{ potencia instalada de Itumbiara - RO. } \\
\cline { 2 - 4 } \multicolumn{1}{c|}{} & \multicolumn{3}{c|}{ Energia Firme - MW.mês } \\
& Sistema & Dif. Ref. & Dif. Incr. \\
\hline Sist. Referência & 10395,4 & - & - \\
\hline$P_{\text {inst }}=1700 M W$ & 11546,3 & 1150,9 & - \\
\hline$P_{\text {inst }}=2100 M W$ & 11546,8 & 1151,5 & 0,4 \\
\hline$P_{\text {inst }}=2500 M W$ & 11546,8 & 1151,5 & 0,0 \\
\hline
\end{tabular}

Tabela 7 - Energia Firme do sistema em função da potência instalada de São Simão - RP.

\begin{tabular}{|l|r|c|c|}
\cline { 2 - 4 } \multicolumn{1}{c|}{} & \multicolumn{3}{c|}{ Energia Firme - MW.mês } \\
\cline { 2 - 4 } \multicolumn{1}{c|}{ Sistema Referência } & 9982,2 & - & Dif. Ref. \\
\hline$P_{\text {inst }}=1400 M W$ & 11106,9 & 1124,7 & Dif. Incr. \\
\hline$P_{\text {inst }}=1680 M W$ & 11298,1 & 1315,9 & 191,2 \\
\hline$P_{\text {inst }}=2200 M W$ & 11352,2 & 1370,0 & 54,1 \\
\hline
\end{tabular}

Tabela 8 - Energia Firme do sistema em função da potência instalada de São Simão - RO.

\begin{tabular}{|l|c|c|c|}
\cline { 2 - 4 } \multicolumn{1}{c|}{} & \multicolumn{3}{c|}{ Energia Firme - MW.mês } \\
\multicolumn{1}{c|}{ Sistema } & Dif. Ref. & Dif. Incr. \\
\hline$P_{\text {inst }}=1400 M W$ & 10212,0 & - & - \\
\hline$P_{\text {inst }}=1680 M W$ & 11545,1 & 1133,1 & - \\
\hline$P_{\text {inst }}=2200 M W$ & 11608,4 & 1334,8 & 201,7 \\
\hline
\end{tabular}

As Tabelas 3 e 4 apresentam os resultados quando o volume máximo de Itumbiara é variado. Comparando os valores das duas tabelas constata-se, de forma semelhante ao ocorrido com Emborcação, que para qualquer volume máximo adotado, a Energia Firme com a RO é sempre maior que com a RP.

Variando o volume máximo de $12700 \mathrm{hm}^{3}$ para $17027 \mathrm{hm}^{3}$, incrementa-se o benefício energético de 118,8 MW.mês com a RP e de 131,6 MW.mês com a RO. Há uma diferença de cerca de $11 \%$ entre os benefícios incrementais. 
Para a variação entre os volumes de $17027 \mathrm{hm}^{3}$ e 21350 $\mathrm{hm}^{3}$, se o sistema é operado com a RP, o benefício energético incremental é de 97,7 MW.mês, enquanto que se a regra adotada for a RO o mesmo benefício é de 139,1 MW.mês. Neste caso a diferença é de $42 \%$.

\subsection{Variações de Potência Instalada}

A análise das variações de potência instalada é realizada mantendo-se o volume máximo das usinas constantes e iguais a seus valores reais.

As Tabelas 5 e 6 mostram os resultados das simulações para cálculo da Energia Firme variando-se a potência instalada em Itumbiara. Os resultados mostram que os ganhos de Energia Firme gerados pela inserção de Itumbiara no SR não se alteraram em função das diferentes potências instaladas adotadas. Isso ocorre porque a potência instalada mínima utilizada para Itumbiara já é capaz de turbinar as vazões afluentes do Período Crítico. Quando mais geradores são instalados, eles simplesmente ficam parados durante o Período Crítico.

Por outro lado, as alterações de potência instalada influenciam de forma diferenciada a Energia Secundária do sistema, já que com a existência de mais máquinas, a usina pode turbinar picos de vazão que outrora seriam vertidos. Este fato mostra os erros que podem ocorrer se somente a Energia Firme for considerada como benefício energético.

As Tabelas 7 e 8 apresentam as variações de Energia Firme do sistema em função da potência instalada da usina de São Simão. Os resultados mostram que para São Simão o aumento da potência instalada produz acréscimos de Energia Firme. Isto ocorre porque esta usina possui uma quantidade de máquinas menor que a necessária para turbinar as vazões afluentes do Período Crítico. Logo, conforme mais máquinas vão sendo adicionadas, mais energia pode ser gerada durante o Período Crítico, aumentando o valor da Energia Firme.

Comparando os ganhos proporcionados pelas diferentes regras constata-se mais uma vez que a RO proporciona valores mais elevados de Energia Firme e maiores benefícios incrementais para todos as potências instaladas analisadas.

\subsection{Quedas das Turbinas}

Na Figura 9 mostra-se a curva de permanência da queda líquida de Itumbiara em duas situações distintas: uma para operação com a RP e outra com a RO. O volume máximo da usina e sua potência instalada correspondem aos seus valores reais.

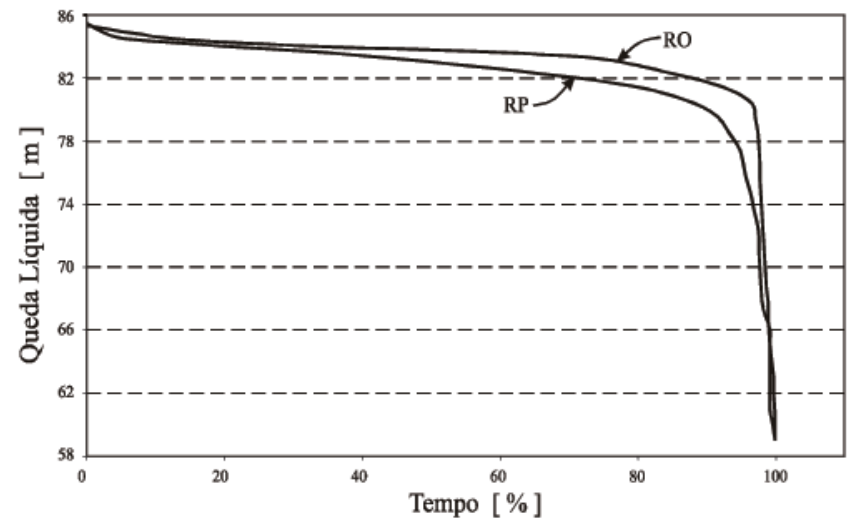

Figura 9 - Curvas de permanências da queda líquida de Itumbiara para a RP e para a RO.

De acordo com a figura, a operação com diferentes Regras de Operação altera a distribuição de queda líquida da usina. No exemplo, a RO garante quedas líquidas maiores que a RP na maior parte do tempo. Conseqüentemente, a operação com a RO garante que as turbinas serão capazes de fornecer potências maiores ao gerador durante períodos mais longos.

Sem considerar qual regra proporciona maior benefício energético, este gráfico mostra a importância das quedas de referência e de projeto da turbina serem projetadas em consonância com a política de operação. Desconsiderações da política de operação podem fazer com que a turbina limite a operação do gerador na maior parte do tempo, subutilizando-se o investimento realizado na compra dos equipamentos elétricos e do próprio gerador.

O contrário também pode acontecer quando a queda de referência de uma turbina é inferior à queda mínima na qual ela opera a maior parte do tempo. Neste caso, o potencial hidroelétrico do rio é subaproveitado, pois a turbina não é capaz de aproveitar as vazões em quedas elevadas, sendo obrigada a fechar seu distribuidor e limitar o fluxo de água.

De forma similar, estimar uma queda de projeto admitindo que a usina operará segundo uma regra e, após sua construção, operá-la com outra, pode significar que a turbina vai trabalhar a maior parte do tempo com rendimento baixo, com altura de queda líquida muito diferente da sua queda de projeto.

\section{CONCLUSÕES}

Este artigo apresentou um modelo de dimensionamento de usinas hidroelétricas que combina um Algoritmo Genético e uma ferramenta de simulação da operação de sistemas hidroelétricos de potência.

Mostrou-se que através deste modelo as possíveis soluções podem ser exploradas com maior eficiência que nas buscas convencionais tradicionalmente utilizadas. Além disso, a 
ferramenta de simulação permite a utilização de diferentes políticas de operação para as usinas, viabilizando assim o dimensionamento de aproveitamentos segundo diferentes formas de operar o sistema.

Com o estudo de caso, mostrou-se que os benefícios energéticos de uma usina hidroelétrica são sensivelmente influenciados pela política de operação utilizada nas simulações.

Testes futuros pretendem avaliar o desempenho global do modelo, através do dimensionamento completo de usinas isoladas e de conjuntos de usinas hidroelétricas.

\section{AGRADECIMENTOS}

Os autores gostariam de agradecer os apoios da Comissão Fulbright, da Cornell University, da FAPESP - Fundação de Amparo à Pesquisa do Estado de São Paulo, e da CAPES - Coordenação de Aperfeiçoamento de Pessoal de Nível Superior. Os autores também agradecem as sugestões e a ajuda do Prof. Dr. Dorel Soares Ramos da Escola Politécnica da Universidade de São Paulo.

\section{REFERÊNCIAS BIBLIOGRÁFICAS}

Army, Department of the Army, Corps of Engineers (1985). Engineering and designing hydropower. Washington, D.C., USA.

Bettega, R. \& F. Ramos (2001). O impacto da comercialização no mercado spot de energia na análise de viabilidade de hidrelétricas. Revista Brasileira de Recursos Hídricos, 6(1): 43-70.

Carneiro, A. A. F. M. \& S. Soares (1993). Reservoir operation rules for hydroelectric power system optimization. IEEE/NTUA Athens Power Tech Conference: "Planning, Operation and Control of Today's Electric Power Systems", Athens, Greece: 965-969.

Carvalho, M. F. \& S. Soares (1987). An efficient hydrothermal scheduling algorithm. IEEE Transactions on Power Systems, PWRS-2(3): 537-542.

CESP, Companhia Energética de São Paulo (1988). Aproveitamento hidroelétrico do rio Ribeira: estudos de inventário das usinas de Divisa, Tijuco, Itaoca, Funil e Batatal. São Paulo, SP.

Eletrobrás, Centrais Elétricas Brasileiras S.A. (1997). SINV 3.0 Programa de Estudos Energéticos. http://www.eletrobras.gov.br/downloads/atuacao/recursos/ sinv3.0.zip, 26/12/2001.

Fortunato, L. A. M., T. A. Araripe Neto, et al. (1990). Introdução ao planejamento da expansão e operação de sistemas de produção de energia elétrica, EDUFF Editora Universitária, Rio de Janeiro, RJ, 232 p.

Gen, M. \& R. Cheng (2000). Genetic algorithms and engineering optimization, Wiley, New York, xvi, 495 p.
Goldberg, D. E. (1989). Genetic algorithms in search, optimization, and machine learning, Addison-Wesley Pub. Co., Reading, Mass., xiii, 412 p.

Horstmann, C. S. (1997). Practical object-oriented development in C++ and Java, Wiley Computer Pub., New York, xiii, 562 p.

Loucks, D. P., J. R. Stedinger, et al. (1981). Water resource systems planning and analysis, Prentice-Hall, Englewood Cliffs, N.J., xv, 559 p.

Silva Filho, D. \& A. A. F. M. Carneiro (1998). Regras otimizadas de operação de sistemas hidroelétricos obtidas por Lógica Fuzzy. XII CBA - Congresso Brasileiro de Automática, Uberlândia, MG, 4: 1351-1356.

Silva Filho, D., A. A. F. M. Carneiro, et al. (2000). Influência das regras de operação sobre parâmetros de dimensionamento de usinas hidroelétricas. VII SEPOPE - Symposium of Specialists in Electric Operational and Expansion Planning, Curitiba, PR, CD-ROM.

Sinha, A. K., B. V. Rao, et al. (1999). Yield model for screening multipurpose reservoir systems. Journal of Water Resources Planning and Management, November/December: 325-332.

Stedinger, J. R., B. F. Sule, et al. (1983). Multiple reservoir system screening models. Water Resources Research, 19(6): 1383-1393.

Stroustrup, B. (2000). The C++ programming language, AddisonWesley, Boston, $\mathrm{x}, 1019 \mathrm{p}$. 